\title{
Advantage of transurethral resection with narrow band imaging for non-muscle invasive bladder cancer
}

\author{
KOHEI KOBATAKE, KOJI MITA, SHINYA OHARA and MASAO KATO \\ Department of Urology, Hiroshima City Asa Hospital, Hiroshima 731-0293, Japan
}

Received July 3, 2014; Accepted April 17, 2015

DOI: $10.3892 / 01.2015 .3280$

\begin{abstract}
The aim of the present study was to compare the benefits of transurethral resection (TUR) under narrow band imaging (NBI-TUR) and TUR under conventional white light imaging (WLI-TUR) for non-muscle invasive bladder cancer (NMIBC). The study cohort consisted of 135 patients with NMIBC who were followed up for $\geq 1$ year after TUR and who received no additional post-operative treatment. In the WLI-TUR group $(n=78)$, systematic intravesical observation under WLI was followed by a multiple site biopsy (MSB), after which lesions detected as positive findings were resected completely under WLI. In the NBI-TUR group ( $\mathrm{n}=57$ ), similar observation under WLI was followed by systematic intravesical observation under NBI. Following MSB under NBI, TUR was performed for all lesions detected as positive findings under NBI. The sensitivity, specificity, positive-predictive value, negative-predictive value (NPV) and accuracy in the NBI-TUR group were calculated using results from the cystoscopical and pathological examinations of MSB samples under WLI and NBI. The tumor recurrence rate was analyzed in the two groups. Background factors did not differ significantly between the two groups, with the exception of the observation period ( 31.0 vs. 15.0 months; $\mathrm{P}<0.01$ ). The procedure under NBI exhibited significantly higher sensitivity (95.0 vs. $70.0 \% ; \mathrm{P}<0.01)$ and NPV (97.1 vs. $86.8 \% ; \mathrm{P}<0.01)$ compared with the procedure under WLI. The 1-year recurrence rate in the NBI-TUR group was significantly lower than that in the WLI-TUR group (21.1 vs. 39.7\%; $\mathrm{P}=0.016)$. In conclusion, the present study indicated that NBI-TUR is more advantageous than conventional WLI-TUR for patients with NMIBC.
\end{abstract}

\section{Introduction}

Narrow band imaging (NBI) is an optics technology that uses the contrast between two wavelengths of light: Cerulean narrow band light at 390-445 nm and green narrow band light

Correspondence to: Dr Koji Mita, Department of Urology, Hiroshima City Asa Hospital, 2-1-1 Kabe-Minami, Asakita-Ku, Hiroshima 731-0293, Japan

E-mail: mita@plum.ocn.ne.jp

Key words: narrow band imaging, bladder cancer, transurethral resection at $530-550 \mathrm{~nm}(1)$. The relative intensities of the cerulean and green bands are increased, while the intensity of red light is minimized under NBI. The contrast of images of capillaries in the surface layers of mucosal membranes is enhanced and detailed pattern can be visualized. The vasculature appears dark green or black against an almost white normal urothelium, whereas similar lesions appear red on a background of pink normal urothelium under white light imaging (WLI). Thus, the contrast between urothelial carcinomas (UCs) and normal urothelium is enhanced by NBI, allowing for the detection of UCs more easily. This feature is particularly noticeable for small or flat early UCs, which conventional WLI struggles to detect (2).

NBI is useful for the identification and evaluation of early-stage cancer in gastrointestinal endoscopy (3), and the utility of NBI for increasing tumor detection and decreasing recurrence compared with WLI has been shown in non-muscle invasive bladder cancer (NMIBC) (4-9). However, it is unclear if transurethral resection (TUR) using NBI (NBI-TUR) reduces the tumor recurrence rate compared with TUR under conventional WLI (WLI-TUR). Therefore, the aim of the present study was to compare the utility of NBI-TUR with WLI-TUR in patients with NMIBC treated at Hiroshima City Asa Hospital (Hiroshima, Japan).

\section{Patients and methods}

Patients. The study cohort consisted of 135 patients with NMIBC who were followed up for $\geq 1$ year after treatment with TUR at Hiroshima City Asa Hospital between January 2010 and April 2014, and who received no post-operative treatment. NBI-TUR has been performed at this hospital since February 2012. All NBI observations were made using a Visera Elite system (Olympus, Tokyo, Japan). Cases that received post-operative intravesical injections of agents such as Bacillus Calmette-Guérin or anticancer agents, those who underwent a second TUR and those with a history of treatment for upper urinary tract cancer were excluded from the study (Fig. 1). TUR was performed under general anesthesia.

Treatment. In the WLI-TUR group, systematic intravesical observation was performed under WLI, and then a multiple site biopsy (MSB) was performed for the detected tumor lesions and other lesions in at least five areas: The bilateral outer ureteral orifice, the bilateral sidewalls and the posterior 


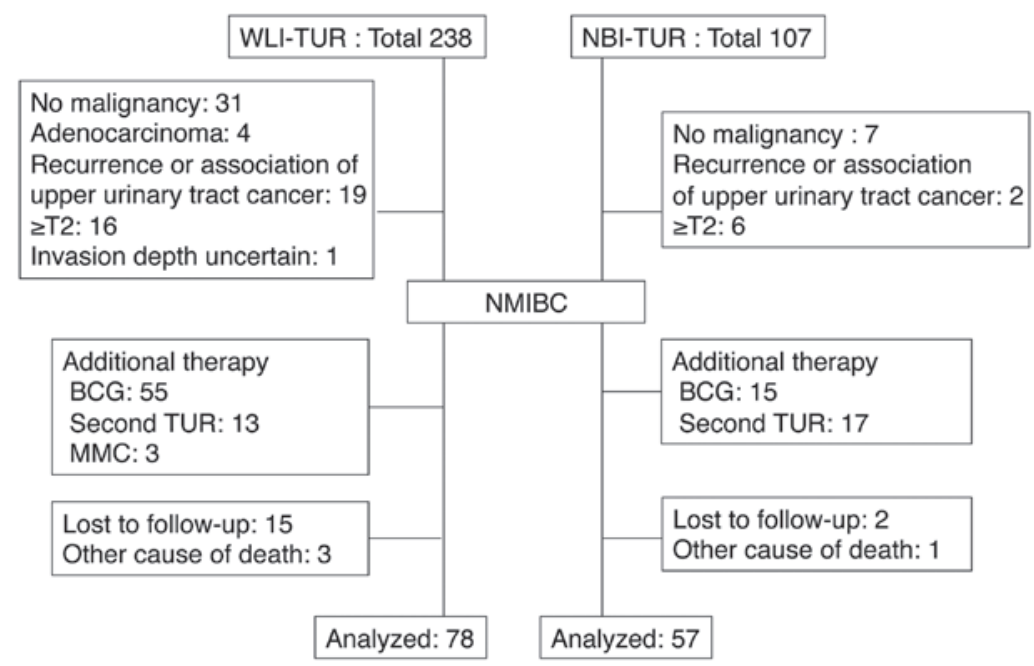

Figure 1. Criteria for patient selection. TUR, transurethral resection; NBI-TUR, TUR under narrow band imaging; WLI-TUR, TUR under white light imaging; BCG, Bacillus Calmette-Guerin; MMC, Mitomycin C; T2, tumor-node-metastasis stage T2.

wall. Lesions with positive findings (abnormal mucosa) were resected completely under WLI.

In the NBI-TUR group, systematic intravesical observation under conventional WLI was conducted using the same procedure as that in the WLI-TUR group and positive findings were recorded. Systematic intravesical observation was then conducted under NBI and positive findings were identified under NBI. MSB as aforementioned was performed under NBI, and TUR was performed completely for all the positive findings under NBI.

Cases with an isolated carcinoma in situ (CIS) lesion were followed up without additional treatment if the lesion was small with positive findings and was resected completely. Other cases, including those difficult to distinguish from normal mucosa or possessing multiple or large lesions, received additional treatment immediately and were not included in this study. The post-operative course was followed in the Outpatient Department by WLI cystoscopy and urinary cytology every three months for all patients. If tumor recurrence was suspected, a pathological examination was performed by biopsy or TUR, and cases with recurrence received immediate additional treatment. Post-operative tumor recurrence was defined as pathological confirmation of UC.

Tumor detection. The tumor detection rates under WLI and NBI were analyzed to determine the sensitivity, specificity, positive-predictive value (PPV), negative-predictive value (NPV) and accuracy of the methods (6). In the NBI-TUR group, these values were calculated separately and compared with the endoscopic findings and the pathological results of MSB under WLI and NBI.

Follow-up. The tumor recurrence rates at 3 months and 1 year after TUR, and the recurrence free-survival rate were analyzed in the NBI-TUR and WLI-TUR groups. To identify independent risk factors for the 1-year recurrence of NBI-TUR, the following background data were analyzed in univariate and multivariate analyses: Age ( $<75$ or $\geq 75$ years old), gender (male or female), tumor history (first or recurrence), tumor number (single or multiple), tumor size ( $<3$ or $\geq 3 \mathrm{~cm}$ ), villous lesion (yes or no), pre-operative urine cytology (negative or positive), pathological stage [non-pathological tumor stage 1 (pT1) or pT1], concomitant CIS (yes or no) and pathological grade (grades 1-2 or 3).

Statistical analysis. Statistical analysis was performed using the $\chi^{2}$ test, unpaired $t$-test, and Mann-Whitney $\mathrm{U}$ test for the patient background factors, the 3-month and 1-year recurrence rates, and the tumor detection rate. Recurrence-free survival was compared using a generalized Wilcoxon test. Logistic multiple regression analysis was used to evaluate items found to be significant in univariate analysis. All statistical analyses were performed using Exel-Toukei 2012 version 1.11 (Social Survey Research Information Co., Ltd., Tokyo, Japan). P<0.05 was considered to indicate a statistically significant difference.

\section{Results}

Background of patients. The backgrounds of the patients in the WLI-TUR $(\mathrm{n}=78)$ and NBI-TUR $(\mathrm{n}=57)$ groups are shown in Table I. Background factors did not differ significantly between the two groups, with the exception of the observation period (31.0 vs. 15.0 months; $\mathrm{P}<0.01)$.

Tumor detection rate under WLI and NBI. In the NBI-TUR group, out of 379 biopsies, CIS was detected in 34 and non-CIS (pT1 or pTa) in 86 . The procedure under NBI exhibited significantly higher sensitivity $(95.0$ vs. $70.0 \%$; $\mathrm{P}<0.01)$ and NPV (97.1 vs. 86.8\%; $\mathrm{P}<0.01)$ compared with the rates under WLI, but the specificity was significantly lower (77.2 vs. $89.7 \%$; $\mathrm{P}<0.01$ ) under NBI. There was no significant difference in PPV and accuracy between NBI and WLI groups (Table II).

There were no cancer lesions identified under WLI that could not be identified under NBI; whereas $41.2 \%$ of CIS and $17.4 \%$ of non-CIS lesions were identified with NBI, but not with WLI; and $14.7 \%$ of CIS and $2.3 \%$ of non-CIS lesions could not be identified under NBI or WLI (Fig. 2). The rate of detection of CIS lesions in the 12 patients with positive 
Table I. Patient background.

\begin{tabular}{|c|c|c|c|}
\hline Parameter & WLI-TUR (n=78) & NBI-TUR (n=57) & P-value \\
\hline Median observation period, months & 31.0 & 15.0 & $<0.001$ \\
\hline Median age, years & 73 & 75 & 0.704 \\
\hline Gender, $\mathrm{n}$ & & & 0.485 \\
\hline Male & 62 & 48 & \\
\hline Female & 16 & 9 & \\
\hline Clinical status, $\mathrm{n}$ & & & 0.999 \\
\hline Newly diagnosed & 33 & 24 & \\
\hline Recurrent (<1 year) & 22 & 16 & \\
\hline Recurrent ( $\geq 1$ year) & 23 & 17 & \\
\hline Number of tumors & & & 0.749 \\
\hline Single & 35 & 24 & \\
\hline Multiple & 43 & 33 & \\
\hline Associated villous lesions, $\mathrm{n}$ & 36 & 31 & 0.345 \\
\hline Tumor size, $\mathrm{n}$ & & & 0.379 \\
\hline$\geq 3 \mathrm{~cm}$ & 9 & 4 & \\
\hline$<3 \mathrm{~cm}$ & 69 & 53 & \\
\hline TNM Stage, $\mathrm{n}$ & & & 0.961 \\
\hline $\mathrm{pTa}$ & 61 & 45 & \\
\hline pT1 & 12 & 9 & \\
\hline pTis & 5 & 3 & \\
\hline Concomitant CIS, n & 11 & 13 & 0.165 \\
\hline Grade, $\mathrm{n}$ & & & 0.436 \\
\hline 1 & 8 & 3 & \\
\hline 2 & 51 & 36 & \\
\hline 3 & 19 & 18 & \\
\hline Urinary cytology (pre-TUR), n & & & 0.780 \\
\hline Negative & 60 & 45 & \\
\hline Positive & 18 & 12 & \\
\hline
\end{tabular}

CIS, carcinoma in situ; pT, pathological tumor stage; NBI, narrow band imaging; WLI, white light imaging; TUR, transurethral resection.

Table II. Tumor detection rate under WLI and NBI. ${ }^{\mathrm{a}}$

\begin{tabular}{lcrr}
\hline Parameter & NBI & WLI & P-value \\
\hline Sensitivity & $95.0 \%(114 / 120)$ & $70.0 \%(84 / 120)$ & $<0.001$ \\
Specificity & $77.2 \%(203 / 263)$ & $89.7 \%(236 / 263)$ & $<0.001$ \\
PPV & $65.5 \%(114 / 174)$ & $75.7 \%(84 / 111)$ & 0.069 \\
NPV & $97.1 \%(203 / 209)$ & $86.8 \%(236 / 272)$ & $<0.001$ \\
Accuracy & $83.6 \%(317 / 379)$ & $84.4 \%(320 / 379)$ & 0.766 \\
\hline
\end{tabular}

${ }^{a}$ Total number of biopsy sites, 379. Total number of UCs, 120 (CIS, 34; non-CIS, 86). Sensitivity, number of cancer lesions in positive findings / total number of cancer lesions; specificity, number of non-cancer lesions in negative findings / total number of non-cancer lesions; positive predictive value (PPV), number of cancer lesions in positive findings / total number of positive findings; negative predictive value (NPV), number of non-cancer lesions in negative findings / total number of negative findings; accuracy, (number of cancer lesions in positive findings + number of non-cancer lesions in negative findings) / total number of biopsies.

urinary cytology was higher than that in the 45 patients with negative urinary cytology $[14.8 \%(12 / 81)$ vs. $7.5 \%(22 / 293)$ biopsies; $\mathrm{P}=0.043$ ].
To evaluate the surgeon's skill in the detection of UCs in NBI, the patients in the NBI-TUR group were divided into chronological groups: The first half $(n=29)$ that 
Table III. Predictors of 1-year recurrence in the transurethral resection under narrow band imaging group.

\begin{tabular}{|c|c|c|c|}
\hline \multirow[b]{2}{*}{ Possible predictor } & \multirow{2}{*}{$\frac{\text { Univariate analysis }}{\text { P-value }}$} & \multicolumn{2}{|c|}{ Multivariate analysis } \\
\hline & & P-value & HR (95\% CI) \\
\hline Recurrent tumor & 0.489 & & \\
\hline Multiple tumors & 0.015 & 0.120 & $6.17(0.62-61.01)$ \\
\hline Tumor size $(\geq 3 \mathrm{~cm})$ & 0.840 & & \\
\hline Associated villous lesion & 0.023 & 0.313 & $2.54(0.42-15.50)$ \\
\hline pT1 & 0.925 & & \\
\hline CIS & 0.238 & & \\
\hline Grade 3 & 0.122 & & \\
\hline Urinary cytology (positive) & 0.005 & 0.037 & $5.24(1.11-24.87)$ \\
\hline Age $(\geq 75$ years $)$ & 0.273 & & \\
\hline Gender (male) & 0.925 & & \\
\hline
\end{tabular}

CIS, carcinoma in situ; HR, hazards ratio; CI, confidence interval; pT1, pathological tumor stage 1.
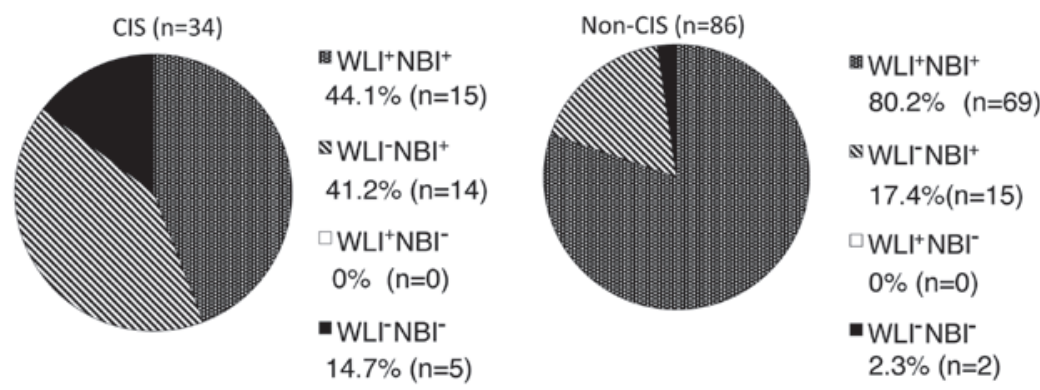

Total number of biopsies $=379$. Total number of detected carcinomas $=120(\mathrm{ClS}=34$, non- $\mathrm{CIS}=86)$

WLI+ or WLI'; Macroscopic findings were positive (visible) or negative (not visible) in WLI. $\mathrm{NBI}^{+}$or $\mathrm{NBI}^{\prime}$; Macroscopic findings were positive (visible) or negative (not visible) in NBI.

Figure 2. Pathological classification and macroscopic findings of tumors detected by multiple site biopsy in the NBI-TUR group. TUR, transurethral resection; NBI-TUR, TUR under narrow band imaging; WLI, white light imaging; CIS, carcinoma in situ .

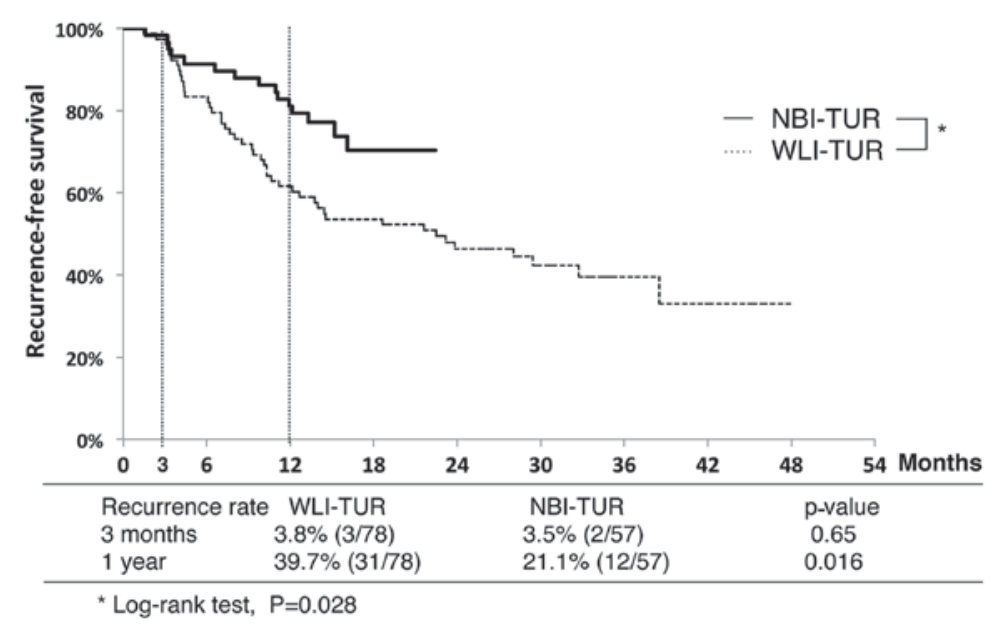

Figure 3. Comparison of recurrence-free survival without additional treatment between the WLI-TUR and NBI-TUR groups. TUR, transurethral resection; NBI-TUR, TUR under narrow band imaging; WLI-TUR, TUR under white light imaging.

underwent the procedure and the second half $(n=28)$. Specificity (68.6 vs. $84.5 \%$; $\mathrm{P}<0.01)$ and accuracy $(80.3$ vs. $87.1 \%$; $\mathrm{P}=0.022)$ were significantly higher in the second group, but sensitivity did not differ significantly between the two groups.
Recurrence rate following TUR. Recurrence-free survival curves for the NBI-TUR and WLI-TUR groups are shown in Fig. 3. The 3-month recurrence rates did not differ between the NBI-TUR $(3.5 \%)$ and WLI-TUR $(3.8 \%)$ groups, but the 
1-year recurrence rate was significantly lower in the NBI-TUR group (21.1 vs. 39.7\%; $\mathrm{P}=0.016$ ). The recurrence-free survival rate was significantly higher in the NBI-TUR group $(\mathrm{P}=0.028)$.

Univariate and multivariate analyses were performed to identify factors associated with 1-year recurrence in the NBI-TUR group. Multiple tumors, an associated villous lesion and positive urinary cytology were significant in univariate analysis, while positive urinary cytology was the only significant predictor in multivariate analysis (Table III).

\section{Discussion}

To the best of our knowledge, the present study is the first to show that NBI-TUR significantly reduces the tumor recurrence rate compared with WLI-TUR in Japanese patients with NMIBC. Moreover, the results suggested that NBI was effective for the detection of CIS lesions, as well as for non-CIS lesions (Fig. 2). There have been few previous comparisons of recurrence in NMIBC following NBI-TUR and WLI-TUR (4). The significantly lower recurrence rate with NBI-TUR compared to WLI-TUR may be due to the significantly higher tumor detection rate with NBI-TUR found in the NMIBC patients in the present study and in previous studies (4-9).

When performing NBI-TUR for all positive findings under NBI, the potential for unnecessary injury should be considered for a non-carcinoma lesion due to the higher false-positive rate found with NBI in the present study. However, based on the present results, such injury can be decreased by the experience of the surgeon, as the specificity was significantly improved in later cases in the NBI-TUR group. Specificity and accuracy in the NBI-TUR group were significantly improved by experience, but the specificity only reached $84.5 \%$ in the later cases and certain lesions, including CIS, could not be detected under NBI. Furthermore, bleeding or active inflammation of the bladder means that the detection of abnormal lesions using NBI is difficult, as enhanced images are generated by light emission from target areas in two narrow wavebands that are strongly absorbed by hemoglobin (7). These results indicate the current technical limitations of NBI.

The present study included cases of small CIS lesions with positive findings that were resected completely. A CIS lesion was not found to be a predictor of 1-year recurrence in the NBI group, but positive urinary cytology was a significant predictor of 1-year recurrence. Positive urinary cytology is important in the diagnosis and follow-up of CIS as it has high sensitivity and specificity for CIS (>90\%) (10). Based on these findings, additional treatment should be administered in all cases with positive urinary cytology and CIS lesions, even if the lesion is small under NBI observation.

The tumor recurrence rate at 3 months after TUR is dependent on the quality of the initial TUR and is an important prognostic factor for recurrence, progression and survival. The tumor recurrence rate at 3 months after TUR in NMIBC may also reflect the skill of the surgeon $(4,11,12)$. Without additional post-operative treatment, recurrence rates of 3.4 to $20.6 \%$ and 7.4 to $45.8 \%$ have been found in patients with single and multiple tumors, respectively (11). Thus, the 3-month recurrence rate after TUR in the present study was not inferior to those of previous studies. These results indicate that the skills of the surgeons in TUR at Hiroshima City Asa Hospital are sufficient to provide confidence in the overall findings of the study.

This study has several limitations, including the retrospective design and the relatively small number of subjects. The treatment strategy for patients with NMIBC basically used the policy in the European Organization for Research and Treatment of Cancer recurrent risk classification $(10,13)$, however, certain high-risk patients in the present study were not willing to undergo additional post-operative treatment, or non-treatment follow-up was recommended due to their physical status.

The detection rate of bladder cancer may be improved by fluorescence cystoscopy using 5-aminolevulinic acid (5-ALA) or hexaminolevulinate, in addition to NBI. In a randomized prospective study of treatment outcome, the tumor-free recurrence rate at 1-year after TUR using 5-ALA was 20\% lower than that after WLI-TUR (14). By contrast, no difference was found in the long-term recurrence- and progression-free rates following TUR using 5-ALA and WLI-TUR (15).

An international multicenter cooperative study comparing the treatment outcomes of NBI-TUR and WLI-TUR is currently in progress (16). The medical costs from diagnosis to mortality for a patient with bladder cancer are high, and any intervention that reduces these costs would be hugely beneficial (17). However, the cost benefit of NBI-TUR compared with WLI-TUR remains unclear (12). If the surgical outcome after NBI-TUR includes reduced post-operative treatment, this procedure will be beneficial to mitigate the burden on patients with NMIBC (5). Thus, it is noteworthy that the present study showed a significantly lower short-term recurrence rate after NBI-TUR than after WLI-TUR for patients with NMIBC. Further analysis is required to examine the longer term effects of NBI-TUR on the recurrence rate. However, the short-term results of the present study suggest that NBI-TUR is advantageous over conventional WLI-TUR in patients with NMIBC.

\section{Acknowledgements}

The present work was presented at the 32nd World Congress of Endourology.

\section{References}

1. Olympus, News release, December 26, 2006, Endoscopic videoscope system: http://www.olympus-europa.com/medical/ en/medical_systems/applications/urology/bladder/narrow_ band_imaging_nbi_/narrow_band_imaging_nbi_html (Accessed April 22, 2015).

2. Hirata I, Nakagawa Y, Ohkubo M, Yahagi N and Yao K: Usefulness of magnifying narrow-band imaging endoscopy for the diagnosis of gastric and colorectal lesions. Digestion 85: 74-79, 2012.

3. Bryan RT, Billingham LJ and Wallace DM: Narrow-band imaging flexible cystoscopy in the detection of recurrent urothelial cancer of the bladder. BJU Int 101: 702-706, 2008.

4. Naselli A, Introini C, Timossi L, et al: A randomized prospective trial to assess the impact of transurethral resection in narrow band imaging modality on non-muscle-invasive bladder cancer recurrence. Eur Urol 61: 908-913, 2012.

5. Cauberg EC, Mamoulakis C, de la Rosette JJ and de Reijke TM: Narrow band imaging-assisted transurethral resection for non-muscle invasive bladder cancer significantly reduces residual tumor rate. World J Urol 29: 503-509, 2011. 
6. Naselli A, Introini C, Bertolotto F, Spina B and Puppo P: Narrow band imaging for detecting residual/recurrent cancerous tissue during second transurethral resection of newly diagnosed non-muscle-invasive high-grade bladder cancer. BJU Int 105: 208-211, 2010.

7. Tatsugami K, Kuroiwa K, Kamoto T, et al: Evaluation of narrow-band imaging as a complementary method for the detection of bladder cancer. J Endourol 24: 1807-1811, 2010.

8. Cauberg EC, Kloen S, Visser M, et al: Narrow band imaging cystoscopy improves the detection of non-muscle-invasive bladder cancer. Urology 76: 658-663, 2010.

9. Herr HW and Donat SM: A comparison of white-light cystoscopy and narrow-band imaging cystoscopy to detect bladder tumour recurrences. BJU Int 102: 1111-1114, 2008.

10. Babjuk M, Oosterlinck W, Sylvester R, Kaasinen E, Böhle A, Palou-Redorta J; European Association of Urology (EAU): EAU guidelines on non-muscle-invasive urothelial carcinoma of the bladder. Eur Urol 54: 303-314, 2008

11. Brausi M, Collette L, Kurth K, van der Meijden AP, et al EORTC Genito-Urinary Tract Cancer Collaborative Group: Variability in the recurrence rate at first follow-up cystoscopy after TUR in stage Ta T1 transitional cell carcinoma of the bladder: A combined analysis of seven EORTC studies. Eur Urol 41: 523-531, 2002.
12. Brausi M: Are new technologies for detection and treatment of non-muscle-invasive bladder cancer (NMIBC) so important? A plea for adoption by teaching programs to improve results after standard white light transurethral resection of NMIBC. Eur Urol 61: 914-916, 2012.

13. Sylvester RJ, van der Meijden AP, Oosterlinck W, et al: Predicting recurrence and progression in individual patients with stage Ta T1 bladder cancer using EORTC risk tables: a combined analysis of 2596 patients from seven EORTC trials. Eur Urol 49: 466-477, 2006.

14. Daniltchenko DI, Riedl CR, Sachs MD, et al: Long-term benefit of 5-aminolevulinic acid fluorescence assisted transurethral resection of superficial bladder cancer: 5-year results of a prospective randomized study. J Urol 174: 2129-2133, 2005.

15. Schumacher MC, Holmäng S, Davidsson T, Friedrich B, Pedersen J and Wiklund NP: Transurethral resection of non-muscle-invasive bladder transitional cell cancers with or without 5-aminolevulinic acid under visible and fluorescent light: results of a prospective, randomised, multicentre study. Eur Urol 57: 293-299, 2010.

16. Clinical Research Office of the Endourological society, The Global Randomized NBI Bladder Cancer study: http://www.croesoffice.org/OngoingProjects/NBIStudy.aspx

17. Botteman MF, Pashos CL, Radaelli A, Laskin B and Hauser R: The health economics of bladder cancer: A comprehensive review of the published literature. Pharmacoeconomics 21: 1315-1330, 2003. 